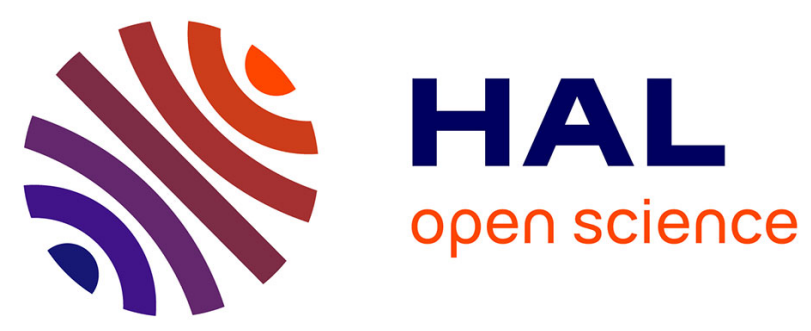

\title{
GENÈSE DU RISQUE ÉDITORIAL : LA COMPTABILITÉ À L'ÉPOQUE ROMANTIQUE DANS LE FONDS DES LIBRAIRES DU COMMERCE
}

Luc Marco

\section{- To cite this version:}

Luc Marco. GENÈSE DU RISQUE ÉDITORIAL: LA COMPTABILITÉ À L'ÉPOQUE ROMANTIQUE DANS LE FONDS DES LIBRAIRES DU COMMERCE. Revue management \& avenir, 2011, Management et Avenir, 1 (41), p. 124-139. 10.3917/mav.041.0124 . hal-01335616

\section{HAL Id: hal-01335616 https://hal.science/hal-01335616}

Submitted on 25 Jun 2016

HAL is a multi-disciplinary open access archive for the deposit and dissemination of scientific research documents, whether they are published or not. The documents may come from teaching and research institutions in France or abroad, or from public or private research centers.
L'archive ouverte pluridisciplinaire HAL, est destinée au dépôt et à la diffusion de documents scientifiques de niveau recherche, publiés ou non, émanant des établissements d'enseignement et de recherche français ou étrangers, des laboratoires publics ou privés. 


\title{
GENÈSE DU RISQUE ÉDITORIAL : LA COMPTABILITÉ À L'ÉPOQUE ROMANTIQUE DANS LE FONDS DES LIBRAIRES DU COMMERCE
} Luc Marco

\author{
Management Prospective Ed. | « Management \& Avenir »
}

2011/1 n $41 \mid$ pages 124 à 139

ISSN 1768-5958

Article disponible en ligne à l'adresse :

http://www.cairn.info/revue-management-et-avenir-2011-1-page-124.htm

\section{!Pour citer cet article :}

Luc Marco, «Genèse du risque éditorial : la comptabilité à l'époque romantique dans le fonds des libraires du commerce », Management \& Avenir 2011/1 ( $\left.{ }^{\circ} 41\right)$, p. 124-139.

DOI 10.3917/mav.041.0124

Distribution électronique Cairn.info pour Management Prospective Ed..

(C) Management Prospective Ed.. Tous droits réservés pour tous pays.

La reproduction ou représentation de cet article, notamment par photocopie, n'est autorisée que dans les limites des conditions générales d'utilisation du site ou, le cas échéant, des conditions générales de la licence souscrite par votre établissement. Toute autre reproduction ou représentation, en tout ou partie, sous quelque forme et de quelque manière que ce soit, est interdite sauf accord préalable et écrit de l'éditeur, en dehors des cas prévus par la législation en vigueur en France. Il est précisé que son stockage dans une base de données est également interdit. 


\title{
Genèse du risque éditorial : la comptabilité à l'époque romantique dans le fonds des libraires du commerce
}

\author{
par Luc Marco 55
}

\author{
Résumé
}

La vente de livres comptables a concerné trois libraires parisiens au XIXe siècle : Bailleul, Renard et Guillaumin. A partir de ce fonds, nous calculons le risque éditorial de cette spécialité entre 1823 et 1850 . Avec une durée moyenne de 25,7 années, l'écoulement de ces livres était très lent. Mais il rendait l'entreprise rentable. Les conditions techniques de l'époque exigeaient un tirage moyen important pour couvrir des prix de revient et des frais généraux élevés.

Abstract

The sale of account books concerned three Parisian booksellers in the XIXth century: Bailleul, Renard, and Guillaumin. From this collection, we calculate the editorial risk of this specialty between 1823 and 1850 . With an average duration of 25.7 years the flow of these books was very slow, but it returned the profitable company. The technical conditions of time required an average mattering edition to cover cost prices and high overheads.

La première définition du risque éditorial a été donnée dès 1763 par le philosophe Denis Diderot dans sa longue lettre sur le commerce de la librairie : « un fonds de librairie est donc la possession d'un nombre plus ou moins considérable de livres propres à différents états de la société, et assorti de manière que la vente sûre mais lente des uns, compensée avec avantage par la vente aussi sûre mais plus rapide des autres, favorise l'accroissement de la première possession » (Diderot, 1763 , p. 12). Le mot de « librairie » désignait à l'époque aussi bien la vente par un imprimeur, un libraire ou un éditeur : les trois fonctions étaient indistinctes sous l'Ancien régime. Le risque éditorial réside dans la durée plus ou moins longue entre les avances de l'édition et les rentrées de la vente en magasin. Pour son temps, Diderot estimait cette durée moyenne à environ dix ans (idem, p. 52). Donc un livre qui est épuisé en moins d'une décennie rapporte de l'argent à la fois à l'éditeur, à l'imprimeur et au libraire ; tandis qu'un ouvrage qui perdure en stock au-delà de cette durée, devient une source de pertes, de ruine peut-être, d'immobilisation de capitaux et de frais récurrents de stockage. Car il faut bien entreposer les ouvrages quelque part en attendant leur écoulement progressif.

55. Luc MARco, Professeur des Universités, Université Paris 13, CEPN-CNRS, luc.marco@univ-paris13.fr 


\section{Genèse du risque éditorial : la comptabilité à l'époque romantique dans le fonds des libraires du commerce}

Pour maîtriser ce risque éditorial inhérent à l'état de libraire, Diderot conseille d'avoir une bonne comptabilité et de se spécialiser dans des genres d'ouvrages qui se vendent bien, ou de se mettre sur une " niche » négligée par les autres libraires-éditeurs ou les imprimeurs-libraires rivaux. Avant l'invention des bazars vers 1820 (Marco, 2009), la pratique des « soldes » ne semble pas avoir germé dans les crânes des libraires, qui préféraient avoir une politique de "fonds ». Mais des remises étaient déjà accordées aux libraires par les imprimeurs qui voulaient écouler leurs stocks plus rapidement.

Le risque éditorial évoqué par Diderot se décompose donc en deux éléments distincts :

a) Le risque initial d'avances aléatoires que prend l'éditeur ou l'imprimeuréditeur, et quelquefois le libraire-éditeur (en tous les cas celui qui possède un capital préalable pour investir dans l'édition d'un livre nouveau).

b) Le risque résiduel des retours financiers irréguliers qu'assume le seul libraire sur son fonds d'ouvrages disponible en magasin (ou dans un entrepôt attenant). Ce risque peut se transmettre tel quel lors de la reprise ultérieure du fonds ou de l'entreprise lors de la succession du libraire en titre.

Tout ceci est bien connu de la littérature spécialisée sur la gestion éditoriale (Barbier, 1990 ; Robin, 2000). Mais que se passait-il alors pour les livres de comptabilité ? Ce travail vise à répondre à deux grandes questions tout en essayant de mesurer plus précisément ce risque éditorial dual. Qui éditait la comptabilité et la vendait après la loi de 1811 sur la profession de libraire ? (point $\mathrm{n}^{\circ} 1$ ). Comment était géré ce fonds spécialisé que nous avons repéré jusqu'au début du Second Empire? (point $\mathrm{n}^{\circ} 2$ ).

L'intérêt historiographique de ces questions nous paraît double. D'une part il permet de retrouver l'existence d'une filière éditoriale spécifique dont le souvenir s'est totalement effacé de la mémoire des historiens-économistes ${ }^{56}$; et, d'autre part, il utilise des méthodes de l'histoire quantitative, pour évaluer un critère de gestion quotidienne qui porte justement sur la littérature elle-même spécialisée en ces techniques.

Cet effet-miroir nous permettra aussi de conclure à quatre niveaux différents mais complémentaires. Etait-ce un petit ou un grand risque ? Etait-il inférieur ou supérieur au risque actuel ? L'absence de normalisation comptable à l'époque jouait-elle à la hausse sur ce risque ? Enfin la part de la comptabilité était-elle stable ou pas dans le fonds général de l'époque romantique?

56. Ainsi, dans sa courte biographie de Guillaumin, madame Le Van Lemesle (1985) ne parle ni de Renard, ni de Bailleul, ni de l'existence de ce fonds préalable en commerce! De même chez Joseph Garnier (1865), pourtant contemporain du grand libraireéditeur d'économie politique, on ne trouve rien sur eux. 


\section{Les libraires spécialisés à Paris}

Nos trois personnages sont des libraires "de fonds ", à la fois éditeurs de leurs propres ouvrages et vendeurs des livres des autres éditeurs du domaine. Ils se distinguent des libraires " d'assortiment " qui ne proposent que des volumes publiés ailleurs (Parinet, 2006, p. 8, note 1). Ils subissent donc un double risque : interne et externe.

En 1791, la libération des professions commerciales du carcan des corporations de métiers a permis de rendre rentable l'idée de spécialiser une librairie sur ces sujets techniques à destination des négociants libres (Marco et Hernandez, 2008). L'impulsion initiale fut cependant l'œuvre d'un simple imprimeur pendant la période troublée de la Terreur.

\subsection{Antoine Bailleul, l'imprimeur du Journal du Commerce}

Frère du célèbre député, avocat et journaliste Jacques-Charles Bailleul (17621843), qui publia lui-même plusieurs ouvrages économiques ${ }^{57}$, Jean-AntoineGuillaume Bailleul est né le 8 janvier 1762 à Bretteville-Saint-Clair, en SeineMaritime. Eduqué pour être notaire, le jeune Antoine devint imprimeur-libraire à Noyon dans l'Oise en 1786 (Krakovitch, 2008, p. 164).

Arrivé à Paris sous la Terreur de 1793, il installe son imprimerie au 22 de la rue Hautefeuille. II fonde à la fin de 1794 le Journal du Commerce, feuille quotidienne patriotique d'obédience libérale qui est située au 742 de la rue Neuve-Augustin puis au 3 rue Grange Batelière. Son biographe en dit ceci : « le fondateur, élevé dans la pratique du droit, y traitait des questions de droit commercial avec un succès tel que chaque jour des négociants avaient recours à lui dans leurs discussions litigieuses, et acceptaient, comme une sorte d'arbitrage, les solutions données dans son journal » (Quérard, 1842, p. 126).

En 1811 deux événements furent marquants pour lui : d'abord il est breveté imprimeur le $1^{\text {er }}$ avril, ensuite son Journal est fusionné par décret impérial avec le Journal de Paris. Heureusement il obtient la survie d'un supplément intitulé le Bulletin du Commerce, qui redevient le $1^{\mathrm{er}}$ janvier 1815 le Journal du Commerce, et qui fusionnera en juillet 1817 avec Le Constitutionnel, avec comme sous-titre le « Journal du commerce, de politique et de littérature ». Après avoir perdu 60.000 francs, le titre est transformé en Le Commerce, journal des progrès moraux et matériels le 10 mai 1837 (Hatin, 1866, p. 239). Depuis 1820, le sous-titre était devenu le Journal du commerce et de l'industrie. Bailleul semble en avoir assuré

57. Parmi près d'une centaine d'ouvrages, nous avons repéré six livres d'économie publique : Observations sur les finances (1799), brochure de 64 pages publiée chez son frère ; Théorie des institutions sociales (1801), Paris, Moutardier, 148 p. ; De la richesse et de l'impôt (1816), Paris, A. Bailleul, 200 p. ; Agence générale des placemens viagers (1820), Paris, Renard ; Dix lettres (1830), Paris, Renard ; Idées anarchiques (1835), Paris, Renard, 200 p. Cela prouve que Bailleul a entretenu des rapports cordiaux avec Renard jusqu'à la fin de sa vie. 


\section{Genèse du risque éditorial : la comptabilité à l'époque romantique dans le fonds des libraires du commerce}

l'impression jusqu'en 1837. II disposait de deux locaux distincts : l'imprimerie proprement dite située au 8 rue Thibautodé, et la librairie au 71 de la rue SainteAnne (anciennement Helvétius) qu'il transmet à Renard fin 1820, l'imprimerie étant reprise par Jean-Sébastien Cordier par la suite. Bailleul est décédé le 25 mars 1843 à Paris ( $2^{\mathrm{e}}$ arrondissement), neuf jours seulement après la mort de son frère (source : Archives de Paris).

Son œuvre personnelle comprend plusieurs écrits économiques, dont deux périodiques : le Journal des fonctionnaires publics (1796) quotidien imprimé par Marchant, avec Gleizal et Le Vasseur comme rédacteurs ; l'Année du négociant et du manufacturier (1803) dirigée en deux volumes avec son frère. Comme ouvrages on notera : un rapport au Directoire exécutif sur Les entrepreneurs des filatures mécaniques de coton (1795) ; le Tableau complet de la valeur des assignats (1796) brochure de 42 pages qui sera treize fois rééditée ; un autre rapport au Conseil des Cinq-Cents intitulé Observations sur le Rapport fait par le citoyen Boissy d'Anglas relatif aux successions des prêtres déportés (1796) ; un Panorama de Paris (1805) ; une Histoire des triomphes militaires (1808), gros livre de 480 pages ; un Avis sur le Traité de commerce et de navigation de 1786 (1814) ; une Petite dissertation sur la découverte de l'imprimerie (1817); et enfin un Mémoire sur la question posée par l'Académie des Sciences morales et politiques par feu l'abbé Grégoire (1839) ${ }^{58}$.

Il avait deux passions : la franc-maçonnerie et l'étude de l'Antiquité. Sur le premier sujet, il publia plusieurs ouvrages en 1830 et 1839. Sur le second, il traduisit en 1821 le grand livre de l'archéologue allemand Roeppen : Crata Repoa ou Initiations aux anciens mystères des prêtres d'Egypte. Au moment de sa mort, en 1843, il préparait un grand ouvrage sur ces questions. C'est lui qui utilisa le premier l'enseigne "Chez Antoine Bailleul, Imprimeur Libraire du Commerce » vers 1811 et que racheta plus tard son successeur Renard.

\subsection{Pierre Renard, le libraire du Commerce}

Homonyme de l'éditeur littéraire François-Sylvain Renard (1777(?)-1821) ${ }^{59}$, notre personnage s'appelait Pierre Hubert Renard. Plus jeune que Bailleul, il est né le 5 octobre 1772 dans le quartier parisien Saint-Sulpice (source : Archives de Paris). II a 41 ans quand il se marie avec Marie-Jeanne Lemignon-Desmares, âgée de 36 ans, le 31 juillet 1813 (idem). Il est breveté en tant que libraire le 21 juin 1820 (AN F18/1818). Pour obtenir ce parchemin, il soumet aux autorités deux certificats : une attestation de moralité et une attestation de capacité. L'attestation de moralité est signée le 19 mai 1820 par deux commerçants : Jean Guerreau, marchand quincaillier, et Pierre Chapsal, marchand de bronze travaillant dans sa rue. L'attestation de capacité est co-signée le 28 mai 1820 par quatre grands

58. Question ainsi posée : "Pourquoi les peuples avancent-ils plus vite dans les sciences que dans la morale pratique ? ».

59. Le seul livre spécialisé en commerce édité par ce premier éditeur Renard est celui de Pierre Périaux (1808). 
libraires de l'époque : Brunot-Labbé, Déterville, Merlin et Caille de Ravée. Ils attestent que Pierre Hubert Renard a déjà 25 ans d'expérience dans la librairie ancienne et moderne!

II reprend le fonds Bailleul après avoir obtenu ce brevet. On apprend sur la couverture de son premier catalogue, que nous avons daté de la fin de 1823, la nature des documents confectionnés par l'imprimerie du Commerce : « Impression de Prix courans, Cours de change, Factures, Bordereaux, Registres, Circulaires, Mémoires, Tableaux à filets de toute espèce. " (Renard, 1823, p. 1). On comprend alors mieux le modèle économique de l'entreprise initiale de Bailleul : utiliser les fonds réguliers dégagés par l'imprimerie dans ces travaux administratifs pour éditer des ouvrages spécialisés, par exemple en gestion militaire (Quillet, 1803), qui étaient alors vendus dans la librairie. Renard va avoir une autre stratégie : constituer un fonds spécialisé en droit, commerce, voyages, littérature, et d'une science nouvelle : l'économie politique. II s'associe avec Jérôme-Adolphe Blanqui pour devenir l'éditeur de l'Ecole Spéciale de Commerce de Paris en 1825 (Renouard, 1921, p. 75) ${ }^{60}$. Ensuite il se spécialise en comptabilité, finance, jurisprudence commerciale et économie politique (ouvrages classiques et modernes). Quand il met en vente son fonds, le 14 juillet 1849, c'est Guillaumin qui l'achète pour renforcer son propre fonds d'ouvrages économiques ${ }^{61}$. Renard sera démissionnaire de son brevet de libraire le 12 septembre 1854, et il sera remplacé par Armand Jean-Michel Dutacq, le propriétaire ${ }^{62}$ du Constitutionnel ! Renard a dû mourir à la fin des années cinquante ou dans les années $1860^{63}$.

\subsection{Gilbert Guillaumin, l'éditeur des économistes et des commerçants}

Gilbert-Urbain Guillaumin est né à Couleuvre, dans l'Allier, le 14 août 1801. II avait donc 39 ans de moins que Bailleul, et dix-neuf ans que Renard. Orphelin à cinq ans. Il est élevé par un rude oncle marchand de bois (Garnier, 1865, p. 113). Monté à Paris en 1819, il devient commis de quincaillerie puis passe chez un commissionnaire. II se lie d'amitié avec le gendre d'un éditeur : Louis BrissotThivars, né en 1792. A l'instigation de celui-ci et de ses amis libéraux, Guillaumin obtient son brevet de libraire en remplacement d'Hippolyte Baudouin en juin 1830 (Le Van Lemesle, 1985, p. 136 ; voir aussi le catalogue de Baudouin, 1820).

Pourtant un catalogue de 8 pages le donne déjà libraire en 1828 avec comme spécialité la littérature et l'histoire (Guillaumin, 1828). Mais cet axe éditorial ne lui

60. En dehors du règlement intérieur de cette école, il a publié, de Blanqui, les livres suivants : Discours de l'Ecole de Commerce (1827) et Histoire de l'exposition des produits de l'industrie française (1827).

61. Sources : Feuilleton du Journal de la Librairie, samedi 14 juillet $1849, n^{\circ} 28$, p. 156 ; Frégier (1849), p. 9 indique que Renard a plus de 30 ans d'ancienneté, ce qui voudrait dire qu'il avait commencé avant son brevet !

62. Dutacq est administrateur du Constitutionnel et du Pays. Il avait demandé un brevet de libraire en 1853, qui lui avait été refusé car il avait fait deux faillites en 1846 : l'une comme directeur du Vaudeville, célèbre théâtre, l'autre comme administrateur de journaux. Comme il a cependant obtenu un concordat en mars 1848 et qu'il possède une fortune de 150.000 francs, le service l'autorise à reprendre le brevet de P.H. Renard (source AN).

63. Aux Archives de Paris, nous avons trouvé quatre « Pierre Renard », décédés entre octobre 1857 et juin 1866. 


\section{Genèse du risque éditorial : la comptabilité à l'époque romantique dans le fonds des libraires du commerce}

permettait pas de couvrir ses frais, malgré son déménagement rue Vivienne puis au 5 du Passage des Panoramas en juin 1832 (Guillaumin, 1832). II décide alors, vers le début de 1835, de se tourner vers l'économie politique, aidé en cela par ses amis Jérôme-Adolphe Blanqui et Horace Say, fils du grand économiste JeanBaptiste Say. Le 27 janvier 1835, il fonde avec un associé la Société d'édition Guillaumin-Levasseur et compagnie.

Son premier grand chantier est une " encyclopédie du commerçant » dont le premier volume consiste en un Dictionnaire du commerce et des marchandises contenant tout ce qui concerne le commerce de terre et de mer, qui paraît en 1837 pour le volume $1^{\text {er }}$ et en 1839 pour le volume 2 (voir la réédition du $1^{\text {er }}$ volume ; Guillaumin, 1852). Ce livre, signé par plus de 35 auteurs $^{64}$, est coordonné par le jeune éditeur ; il sera profondément remanié en 1859 et réédité en 1863 sous le titre de Dictionnaire du commerce et de la navigation. Suite à la reprise du fonds Renard, fin 1849, sa libraire change donc de nom et devient la Librairie du commerce et de l'économie politique, de Guillaumin et $C^{i e}$. En 1856, il inverse les deux domaines pour mettre l'économie politique avant le commerce (Guillaumin, 1856). Puis progressivement, au fur et à mesure que le fonds Renard s'épuise, il ne conserve que l'appellation "Librairie de Guillaumin et $C^{i e}$. " (voir dans Jules de Vroil, 1870).

Victime d'une crise cardiaque en pleine rue, Guillaumin est mort à 63 ans, le 15 décembre 1864. II a donc repris le flambeau de Renard-Bailleul et publié ensuite plusieurs comptables dont le célèbre Jean Gustave Courcelle-Seneuil (Marco, 1991). Mais qu'y avait-il donc de si important dans le fonds Renard pour que notre éditeur spécialisé en économie politique décidât de l'acheter en vente de gré à gré ?

Nous donnons, dans les trois tableaux ci-après, la liste des ouvrages de comptabilité vendus par ces trois librairies, et la part de leur propre contribution éditoriale sous leurs noms propres ou en "portage " de comptes d'auteurs (appelés aujourd'hui l'autoédition).

Tableau 1 - Liste des ouvrages de comptabilité du fonds Renard-Guillaumin

\begin{tabular}{|l|c|l|l|}
\hline \multicolumn{1}{|c|}{ Auteur } & \multicolumn{1}{c|}{ Période } & \multicolumn{1}{c|}{ Titre } & \multicolumn{1}{c|}{ Editeur } \\
\hline GIRAUDEAU, P. & $1793-1850$ & La banque rendue facile & Impr. A. Leroy, Lyon. \\
\hline GERARD, J. & $1816-1833$ & $\begin{array}{l}\text { Cours élémentaire et pratique de la tenue } \\
\text { des livres en partie double }\end{array}$ & L'auteur, Marseille. \\
\hline GARNIER & $1809-1840$ & Eléments de la tenue des livres & L'auteur, Langres. \\
\hline $\mathrm{B}^{* * *}, \mathrm{M}$. & $1817-1827$ & L'esprit de la tenue des livres & Testu, Paris. \\
\hline
\end{tabular}

64. Liste des économistes associés à cette entreprise libérale : Adolphe Blaise (des Vosges), J.A. Blanqui, H. Dussard, Théodore Fix, Joseph Garnier, Mac Culloch, H. Say. Liste des commerçants : Blay, Cortambert, Legentil, Mignot, Rey. Liste des comptables : Durmont, B. Pance, et Wantzell. 


\begin{tabular}{|c|c|c|c|}
\hline PAYEN, A. & $1817-1859$ & Essai sur la tenue des livres & Johanneau, Paris. \\
\hline DEGRANGE, Ed. & $1819-1833$ & Feuilles gravées & Saintin, Paris. \\
\hline MASQUELIER, H. & $1819-1827$ & Le Guide du parfait commerçant & Delaunay, Paris. \\
\hline $\mathrm{C}^{* * *}$, A. ou V. & $1818-1840$ & Instruction sur une tenue des livres & Renard, Paris. \\
\hline QUINEY, J.S. & $1817-1850$ & Livre de raison & Eberhart, Paris. \\
\hline PAYEN, A. & $1819-1840$ & Méthode de l'actif et du passif & Johanneau, Paris. \\
\hline DELORME, B. & $1808-1850$ & Nouveau système de tenue des livres & Chambeau, Avignon. \\
\hline GONON & $1809-1823$ & $\begin{array}{l}\text { Nouvelle méthode de tenir les livres à } \\
\text { parties doubles }\end{array}$ & $\begin{array}{l}\text { Schwarzengerg, Aix-la- } \\
\text { Chapelle. }\end{array}$ \\
\hline ISLER, J. & $1809-1859$ & Nouvelle méthode suisse & Weissenburg, Bruxelles. \\
\hline DOMENGET, G. & $1809-1850$ & $\begin{array}{l}\text { Nouvelle méthode pour la tenue des } \\
\text { livres }\end{array}$ & Weissenburg, Bruxelles. \\
\hline CHALIER, J. & $1818-1823$ & Précis élémentaire de la comptabilité & Eymery, Paris. \\
\hline ASSELIN, M. & $1821-1874$ & Précis sur la tenue des livres & Eymery, Paris. \\
\hline LEGRET, G.P. & $1811-1874$ & $\begin{array}{l}\text { Rudiment de la comptabilité commerciale } \\
\text { ( } 2^{e} \text { éd. 1828) }\end{array}$ & $\begin{array}{l}\text { Belin fils et Prieur, Paris } \\
\left.\text { ( } 2^{\mathrm{e}} \text { éd. Renard }\right) .\end{array}$ \\
\hline BOUCHER, P. & $1803-1840$ & $\begin{array}{l}\text { La science des négociants et teneurs de } \\
\text { livres }\end{array}$ & Levrault et Schoell, Paris. \\
\hline MIGNERET & $1802-1840$ & La science des négociants & Richard, Paris. \\
\hline DE LA PORTE & $1798-1833$ & La science des négociants & Libraires associés, Paris. \\
\hline DEGRANGE, Ed. & $1819-1864$ & La tenue des livres rendue facile & Saintin, Paris. \\
\hline DEGRANGE, Ed. & $1804-1833$ & Supplément à la tenue des livres & Saintin, Paris. \\
\hline DEGRANGE, E. fils & $1807-1827$ & Tenue des livres en double partie & Hocquart, Paris. \\
\hline DEGRANGE, E. fils & $1809-1840$ & Tenue des livres généralisée & Hocquart, Paris. \\
\hline DEGRANGE, E. fils & $1805-1827$ & Balance générale simplifiée & Hocquart, Paris. \\
\hline BOUCHAIN & $1819-1833$ & Traité pratique de la tenue simplifiée & Impr. Marie, Rouen. \\
\hline BLONDEL & $1801-1833$ & La tenue des livres de commerce & L'auteur, Lyon. \\
\hline BUZENET, J. & $1826-1864$ & Guide du commerce & Bachelier, Paris. \\
\hline COFFY, R.P.A. & $1827-1840$ & $\begin{array}{l}\text { Tableau synoptique des principes } \\
\text { généraux de la tenue des livres à parties } \\
\text { doubles }\left(2^{e} \text { éd.) }\right.\end{array}$ & Renard, Paris. \\
\hline COURTET, Ed. & $1821-1840$ & Cours de tenue de livres & $\begin{array}{l}\text { Tournachon-Molin et } \mathrm{H} \text {. } \\
\text { Séguin, Paris. }\end{array}$ \\
\hline DE LA TASSE, C. & $1825-1864$ & Comptabilité rurale & $\begin{array}{l}\text { Carpentier-Méricourt, } \\
\text { Paris. }\end{array}$ \\
\hline DEZARNAUD, B. & $1825-1874$ & Essai sur la comptabilité commerciale & Philippe, Paris. \\
\hline DROUET, F. & $1824-1833$ & $\begin{array}{l}\text { Les parties doubles réduites à leur plus } \\
\text { simple expression }\end{array}$ & L'auteur, Paris. \\
\hline GODARD, P.-A. & $1827-1874$ & $\begin{array}{l}\text { Traité général et sommaire de la } \\
\text { comptabilité commerciale }\end{array}$ & Renard, Paris. \\
\hline GUENAL & $1823-1827$ & Tenue des livres & Bacheville, Paris. \\
\hline JACQUET, J.-B. & $1826-1833$ & De la tenue des livres à parties doubles & Brissot-Thivars, Paris. \\
\hline LEMOINE, R.J. & $1827-1874$ & Répertoire commercial & Daubrée, Paris. \\
\hline MASQUELIER, H. & $1821-1833$ & $\begin{array}{l}\text { Tenue des livres on ne peut plus } \\
\text { simplifiée }\end{array}$ & Martinet, Paris. \\
\hline RUELLE, J.-R. & $1805-1864$ & L'art de tenir les livres en partie double & Reymann, Lyon. \\
\hline VAUTRO, P.-C.-L. & $1826-1840$ & De la tenue des livres en partie double & Emler, Paris. \\
\hline FEIGNAUX & $1827-1833$ & $\begin{array}{l}\text { Cours théorique et pratique de la tenue } \\
\text { des livres }\end{array}$ & L'auteur, Paris. \\
\hline JEANNIN, M. & $1829-1833$ & Traité de la comptabilité commerciale & Renard, Paris. \\
\hline
\end{tabular}




\section{Genèse du risque éditorial : la comptabilité à l'époque romantique dans le fonds des libraires du commerce}

\begin{tabular}{|c|c|c|c|}
\hline LORIMIER, D.F. & $1827-1874$ & $\begin{array}{l}\text { Essai sur les comptes sociaux en } \\
\text { participation }\end{array}$ & Latour, Paris. \\
\hline MARMET, E.C. & $1833-1840$ & Principes de la tenue des livres & Hachette, Paris. \\
\hline SIMON, N.F. & $1832-1864$ & Méthode complète de la tenue des livres & Cornillac, Paris. \\
\hline ROUPP, R. & $1829-1833$ & Tenue des livres en partie double & Lacroix, Paris. \\
\hline ROUPP, R. & $1832-1833$ & $\begin{array}{l}\text { Théorie de l'infini, ou tenue des livres en } \\
\text { parties triples }\end{array}$ & Vacher frères, Paris. \\
\hline TREMERY, F. & $1833-1840$ & Manuel complet du teneur de livres & Roret, Paris. \\
\hline BELLAY, J. & $1838-1855$ & $\begin{array}{l}\text { Science de l'administration commerciale } \\
\text { (2e édition) }\end{array}$ & Renard, Paris. \\
\hline CLAUDE, S. & $1840-1841$ & Cours complet ( $3^{\ominus}$ édition) & Renard, Paris. \\
\hline DELAFONTAINE, A. & $1834-1850$ & $\begin{array}{l}\text { Manuel raisonné des chefs et commis de } \\
\text { comptoir }\end{array}$ & Renard, Paris. \\
\hline $\begin{array}{l}\text { H A C Q U I N - } \\
\text { LABOUREAU, J. }\end{array}$ & $1840-1859$ & $\begin{array}{l}\text { Manuel général de comptabilité financière, } \\
\text { administrative, civile }\end{array}$ & Renard, Paris. \\
\hline JACLOT, J.J. & $1840-1841$ & $\begin{array}{l}\text { La tenue des livres enseignée en } 21 \\
\text { leçons }\end{array}$ & Audin, Paris. \\
\hline JAFFA, W. & $1840-1841$ & Tenue des livres de commerce & Renard, Paris \\
\hline MALLERANGE, J.M. & $1838-1840$ & $\begin{array}{l}\text { Cours analytique, théorique et pratique de } \\
\text { comptabilité commerciale }\end{array}$ & Renard, Paris. \\
\hline SCHASTEL, L.F. & $1836-1874$ & $\begin{array}{l}\text { Le régulateur des opérations de } \\
\text { commerce }\end{array}$ & Renard, Paris. \\
\hline MADAULE & $1845-1864$ & Nouvelle tenue des livres & Renard, Paris. \\
\hline MERLE, L.-M.-C. & $1841-1874$ & $\begin{array}{l}\text { Traité élémentaire du commerce et des } \\
\text { finances ( } 6^{e} \text { édition) }\end{array}$ & Renard, Paris. \\
\hline QUEVY, P. & $1843-1874$ & $\begin{array}{l}\text { Méthode nouvelle de comptabilité } \\
\text { commerciale et spéciale des marchés à } \\
\text { terme ou à loyer }\end{array}$ & L'auteur, Paris. \\
\hline ROSAZ, S. L. & $1814-1850$ & Arithmétique de commerce & Impr. Ballanche, Lyon \\
\hline
\end{tabular}

Source : Catalogues Renard et Guillaumin. Nous avons stoppé nos dépouillements à l'année 1874 car les catalogues ultérieurs de Guillaumin sont incommunicables à la BNF.

Tableau 2 - Complément d'ouvrages hors rubrique « tenue des livres » dans les catalogues Renard et Guillaumin

\begin{tabular}{|l|l|l|l|}
\hline Auteur & Période & Titre & Editeur \\
\hline GUEPRATTE, F.-J. & $1827-1833$ & $\begin{array}{l}\text { Comptabilité commerciale et } \\
\text { manufacturière en parties doubles, } \\
\text { appropriées aux papeteries et pouvant } \\
\text { s'appliquer à toute fabrication et à tout } \\
\text { commerce }\end{array}$ & Impr. Thiel, Metz. \\
\hline RICARD, S. fils & $1798-1840$ & Traité général du commerce (3 vol.) & Laveaux, Paris. \\
\hline SAVARY, J. & $1777-1840$ & Le parfait négociant & Estienne, Paris. \\
\hline MASQUELIER, H. & $1826-1833$ & Comptabilité des ménages & Hautecœur-Martinet, Paris. \\
\hline MONTCLOUX, H. & $1840-1849$ & De la comptabilité publique & Paul Dupont, Paris. \\
\hline
\end{tabular}

Source : idem. 
Tableau 3 - Part de Renard et Guillaumin dans la production des livres de comptabilité entre 1823 et 1850

\begin{tabular}{|l|c|c|c|}
\hline \multicolumn{1}{|c|}{ Editeurs } & $\begin{array}{c}\text { Production propre } \\
\text { (nombre d'ouvrages) }\end{array}$ & Portage de comptes d'auteur & $\begin{array}{c}\text { Total } \\
\text { (nombre cumulé) }\end{array}$ \\
\hline Renard puis Guillaumin & 14 & 6 & 20 \\
\hline Autres éditeurs & 41 & 4 & 45 \\
\hline Ensemble & 55 & 10 & 65 \\
\hline
\end{tabular}

Source : comptage à partir des tableaux I et II.

\section{Le fonds comptable commun}

A partir des différents catalogues conservés à la Bibliothèque Nationale de France (fonds $Q 10$ ), à ceux retrouvés sur Internet ou étant en notre possession lors de l'achat de livres qui les contenaient en annexe, nous allons essayer de mesurer la part de la comptabilité dans ce fonds Renard, de 1823 à 1840, puis dans le fonds Guillaumin en 1849-1850. L'imprimeur Bailleul n'ayant pas laissé, à notre connaissance, de catalogue, nous limiterons notre étude à la période « romantique » soit entre les années 1820 et les années 1850. Si l'évaluation de Diderot est bonne, le stock Renard dans le catalogue Guillaumin doit disparaître vers 1860 environ $(1849+10)$. S'il a disparu avant c'est un succès éditorial pour ces livres spécialisés à l'écoulement assez lent. S'il n'est pas épuisé à cette date, ce serait un échec relatif.

\subsection{Part dans l'assortiment global}

Au début la comptabilité ne représente seulement que $9 \%$ du fonds Renard, ce taux passant ensuite à $7,8 \%$ en 1827 , puis à $7,1 \%$ en 1840 , et enfin à $12,4 \%$ en 1849-1850 dans le catalogue Guillaumin. Ce taux est à comparer avec les autres rubriques : économie politique, commerce, divers... L'économie politique se développe plus vite que la comptabilité. La catégorie la plus nombreuse est l'ensemble " commerce, industrie, sciences ", devant la catégorie "littérature et ouvrages divers ". Renard était donc un libraire assez éclectique dans son domaine, mais qui avait su flairer l'avenir des sciences commerciales et économiques. Son penchant pour le libéralisme l'aida certainement à détecter les auteurs les plus prometteurs dans ces secteurs d'avenir...

Tableau 4 - Nombre de livres par grandes catégories

\begin{tabular}{|l|c|c|c|c|c|c|}
\hline \multicolumn{1}{|c|}{ Catégorie } & $\mathrm{R}-1823$ & $\mathrm{R}-1827$ & $\mathrm{R}-1833$ & $\mathrm{R}-1840$ & $\mathrm{G}-1849-1850$ & Moyenne \\
\hline Commerce, Industrie, Sciences & 43 & 80 & 134 & 104 & 35 & 79,2 \\
\hline Littérature et ouvrages divers & 70 & 87 & 78 & 69 & 20 & 64,8 \\
\hline Jurisprudence commerciale & 31 & 63 & 87 & 87 & 28 & 59,2 \\
\hline Economie Politique, Finances & 22 & 56 & 71 & 74 & 33 & 51,2 \\
\hline Poids, mesures, monnaies & 31 & 38 & 39 & 38 & 22 & 33,6 \\
\hline Comptabilité (tenue des livres) & 26 & 40 & 37 & 37 & 27 & 33,4 \\
\hline
\end{tabular}




\section{Genèse du risque éditorial : la comptabilité à l'époque romantique dans le fonds des libraires du commerce}

\begin{tabular}{|l|c|c|c|c|c|c|}
\hline Calcul, changes, arbitrages & 20 & 39 & 41 & 43 & 19 & 32,4 \\
\hline Calcul des intérêts & 13 & 29 & 29 & 29 & 19 & 23,8 \\
\hline $\begin{array}{l}\text { Commerce, Administration, } \\
\text { Douanes }\end{array}$ & 17 & 22 & $43^{*}$ & 15 & 15 & 22,4 \\
\hline $\begin{array}{l}\text { Ouvrages par souscription, } \\
\text { Additions }\end{array}$ & 5 & 35 & 16 & 21 & 0 & 15,4 \\
\hline Ouvrages nouveaux & 12 & 23 & 0 & 4 & 0 & 7,8 \\
\hline Total & 290 & 512 & 575 & 521 & 218 & 423,2 \\
\hline
\end{tabular}

Sources : nos dépouillements des catalogues dans le fonds BNF Q 10.

* Vingt ouvrages sont des tarifs étrangers traduits et publiés par le Ministère du Commerce.

La comptabilité est la sixième catégorie en nombres d'ouvrages proposés à la vente, intercalée entre les " poids et mesures » et la rubrique " calcul, changes, arbitrages " qui concerne plus les banquiers et financiers. La catégorie " calcul des intérêts " est cependant proche de la comptabilité, et l'ensemble des trois rubriques forme un ensemble remarquable pour une librairie de ce type à l'époque. En est-il de même pour le classement en valeur?

\subsection{Proportions financières du fonds}

En valeur, la comptabilité pèse seulement $6,1 \%$ du fonds Renard, cette proportion partant de 7,3\% en 1823 pour atteindre 7,9\% dans la partie du fonds reprise par Guillaumin. La comptabilité gagne cependant une place dans le classement général : en cinquième catégorie juste derrière "l'économie politique et la finance », et juste devant « calcul, changes, arbitrages ». C'est toujours l'association tripartite entre le commerce, l'industrie et les sciences, qui est la plus rémunératrice, devant la jurisprudence commerciale qui profite de prix individuels plus élevés pour des livres à la pagination plus importante (suivi de la littérature...). Le prix des livres techniques était alors très élevé pour un particulier, mais semblait plus abordable pour une entreprise qui investissait dans une sorte de « formation permanente » avant la lettre. La réputation de nos trois libraires a eu le temps de s'installer sur le marché parisien, puisque leur activité s'étend sur environ un demi-siècle d'existence.

Tableau 5 - Nombre multiplié par le prix franco de port (en francs)

\begin{tabular}{|l|c|c|c|c|c|c|}
\hline \multicolumn{1}{|c|}{ Catégorie } & $\mathrm{R}-1823$ & $\mathrm{R}-1827$ & $\mathrm{R}-1833$ & $\mathrm{R}-1840$ & $\mathrm{G}-1849-1850$ & Moyenne \\
\hline Commerce, Industrie, Sciences & 446,35 & 849,35 & 1172,35 & 1095,25 & 341,1 & 780,88 \\
\hline Jurisprudence commerciale & 333,25 & 566,95 & 821,00 & 808,0 & 173,35 & 540,51 \\
\hline Littérature et ouvrages divers & 427,55 & 649,07 & 516,35 & 438,45 & 263,5 & 458,98 \\
\hline Economie Politique, Finances & 94,4 & 441,6 & 573,85 & 576,85 & 216,75 & 380,69 \\
\hline Comptabilité (tenue des livres) & 146,0 & 216,75 & 260,25 & 236,25 & 120,25 & 195,9 \\
\hline Calcul, changes, arbitrages & 133,8 & 255,0 & 237,35 & 246,6 & 93,30 & 193,21 \\
\hline $\begin{array}{l}\text { Commerce, Administration, } \\
\text { Douanes }\end{array}$ & 108,35 & 186,8 & 289,90 & 160,25 & 157,75 & 180,61 \\
\hline Poids, mesures, monnaies & 190,25 & 200,75 & 203,00 & 158,75 & 100,25 & 170,60 \\
\hline $\begin{array}{l}\text { Ouvrages par souscription, } \\
\text { Additions }\end{array}$ & 62,0 & 301,5 & 181,50 & 85,75 & 0 & 126,15 \\
\hline
\end{tabular}




\begin{tabular}{|l|c|c|c|c|c|c|}
\hline Calcul des intérêts & 30,75 & 104,2 & 102,55 & 118,8 & 61,35 & 83,53 \\
\hline Ouvrages nouveaux & 40,0 & 208,0 & 0 & 145,0 & 0 & 78,6 \\
\hline Total. ............. & 2012,7 & 3979,97 & 4358,10 & 4069,95 & 1527,6 & 3189,64 \\
\hline
\end{tabular}

Sources : nos dépouillements des catalogues dans le fonds BNF Q 10.

La somme annuelle double en dix ans, passant d'un peu plus de 2.000 francs en 1823, à près de 4.400 francs en 1833. Ce qui donne un prix moyen du livre qui s'élève à 7,58 francs en 1833 et 7,54 francs sur l'ensemble de la période. $A$ noter une baisse notable du prix moyen dans la reprise du fonds par Guillaumin : 7 francs tout ronds. Cela est logique car le fonds repris était vieillissant et le nouveau libraire a dû accorder des remises pour écouler plus vite cet assortiment certes homogène mais plus pratique que son fonds originel (plus tourné vers les économistes théoriciens). Cette baisse a dû avoir une influence sur la durée d'épuisement.

\subsection{Epuisement du stock et profit estimé}

Pour pouvoir estimer l'épuisement du stock en valeur, nous avons besoin d'avoir une estimation du prix de revient global des ouvrages, et du coût de stockage à moyen terme. Sur le premier élément nous disposons des informations données par Fontaine de Resbecq en 1863, qui intègrent le coût de composition, de tirage et de papier. A partir de ses données, nous avons rajouté le coût de fabrication de la couverture et du pelliculage (brochage, glaçage, satinage). André Cochut donne un exemple pour un tirage à 500/1.000 exemplaires, avec les bénéfices associés pour une édition anglaise (Guillaumin, 1839, t. 2, p. 1307).

Tableau 6 - Le prix de revient moyen d'un livre imprimé vers 1860 (en francs).

\begin{tabular}{|l|c|c|c|}
\hline Format & A 1.500 exemplaires & A 3.000 exemplaires & A 6.000 exemplaires \\
\hline In- $8^{\circ}$ papier carré 30 feuilles & 1,5532 & 1,2366 & 1,1083 \\
\hline In-18 jésus 10 feuilles $360 \mathrm{p}$. & 1,0492 & 0,7909 & 0,6843 \\
\hline In-32 jésus 6 feuilles & 0,7829 & 0,5831 & 0,4871 \\
\hline
\end{tabular}

Source : nos calculs d'après Fontaine de Resbecq (1863, p. 365).

Cela veut dire qu'un livre qui est vendu 10 francs franco de port, doit avoir un coût maximum de stockage que l'on peut estimer selon la durée probable de maintien dans l'assortiment ou dans le fonds spécialisé. Nous retiendrons l'évaluation moyenne de Diderot et deux durées plus longue : à 20 et 30 ans. 


\section{Genèse du risque éditorial : la comptabilité à l'époque romantique dans le fonds des libraires du commerce}

Tableau 7 - Le coût de stockage annuel estimé maximum en fonction de la durée de vie du livre et du prix de revient unitaire (en francs)

\begin{tabular}{|c|c|c|c|}
\hline Format et tirage & Epuisé en 10 ans & Epuisé en 20 ans & Epuisé en 30 ans \\
\hline In-8 ${ }^{\circ}$ papier carré 30 feuilles & 0,16 & 0,08 & 0,05 \\
$-\quad 1.500$ ex. & 0,12 & 0,06 & 0,04 \\
$-\quad 3.000$ ex. & 0,11 & 0,06 & 0,04 \\
$-\quad 6.000$ ex. & 0,11 & 0,05 & 0,04 \\
\hline In-18 jésus 10 feuilles 360 p. & 0,08 & 0,04 & 0,03 \\
$-\quad 1.500 \mathrm{ex.}$ & 0,07 & 0,04 & 0,02 \\
\hline$-\quad 3.000 \mathrm{ex}$. & 0,08 & 0,04 & 0,03 \\
$-\quad 6.000 \mathrm{ex}$. & 0,06 & 0,03 & 0,02 \\
\hline In-32 jésus 6 feuilles & 0,05 & 0,02 & 0,02 \\
\hline
\end{tabular}

Source : données du tableau 3 divisées par la durée estimée d'épuisement du livre.

Donc, en multipliant le nombre de livres en assortiment et son estimation en stock, nous pouvons calculer le coût de stockage moyen annuel. En le déduisant du chiffre d'affaires estimé, nous aurons le bénéfice brut pour chacune de nos quatre années de référence. En comparant ensuite l'épuisement mesuré des livres de comptabilité, on pourra avoir une idée de leur profitabilité. Nos calculs sont facilités par l'absence d'inflation ou de déflation sur cette époque de référence, la valeur de la monnaie restant stable. Pour avoir des données plus précises, il faudrait recourir aux archives de nos libraires, qui se trouvent peut-être dans le fonds F 18 des Archives Nationales (rubrique "Imprimerie, librairie, presse, censure »). Ce travail sera réalisé dans une étape ultérieure.

Tableau 8 - La durée d'épuisement des ouvrages de comptabilité (en années)

\begin{tabular}{|l|c|c|c|}
\hline Millésime & Durée moyenne & Livres les + vite vendus & Livres les - vite vendus \\
\hline Stock de 1823 & $29,8(n=26)$ & $9,3(n=4)$ & $53,6(n=5)$ \\
\hline Nouveautés 1827 & $29,2(n=14)$ & $10,5(n=4)$ & $48,2(n=5)$ \\
\hline Nouveautés 1833 & $14,3(n=8)$ & $3,8(n=4)$ & $42,5(n=2)$ \\
\hline Nouveautés 1840-49 & $18,3(n=12)$ & $1,3(n=4)$ & $34,5(n=4)$ \\
\hline Ensemble & $25,7\left(\Sigma_{n}=60\right)$ & $6,2\left(\Sigma_{n}=16\right)$ & $45,8\left(\sum_{n}=16\right)$ \\
\hline
\end{tabular}

Source : nos suivis à partir des catalogues Renard et Guillaumin.

Pour estimer les profits engendrés par la vente de ces livres, nous allons appliquer la formule suivante :

Profit global des ouvrages $=[($ Prix des ouvrages $) \times($ tirage $)]-[($ coût de stockage $) \times($ tirage $) \times$ (durée d'épuisement) $]-[($ Prix de revient) $\times$ (tirage $)]-$ [(Frais généraux) $x$ (durée d'épuisement)] 
Tableau 9 - La profitabilité estimée du fonds de livres comptables in- $8^{\circ}$ (en francs)

\begin{tabular}{|c|c|c|c|}
\hline Millésime & $\begin{array}{l}\text { Profit global } \\
\text { sur la période }\end{array}$ & Livres les + vite vendus* & Livres les - vite vendus* \\
\hline \begin{tabular}{l}
\multicolumn{2}{l}{ Stock de 1823} \\
$-\quad 1.500 \mathrm{ex}$. \\
$-\quad 3.000 \mathrm{ex}$. \\
$-\quad 6.000 \mathrm{ex}$.
\end{tabular} & $\begin{array}{c}35635,2 \\
251914,2 \\
683398,2\end{array}$ & $\begin{array}{c}30662,7 \\
79414,2 \\
159598,2\end{array}$ & $\begin{array}{c}35770,2 \\
74098,2 \\
148966,2\end{array}$ \\
\hline $\begin{array}{cc}\text { Nouveautés } 1827 \\
-\quad 1.500 \mathrm{ex} . \\
-\quad 3.000 \mathrm{ex} . \\
-\quad 6.000 \mathrm{ex} .\end{array}$ & $\begin{array}{c}-73594,8 \\
29836,6 \\
235642,2 \\
\end{array}$ & $\begin{array}{c}27197,7 \\
55660,2 \\
112090,2 \\
\end{array}$ & $\begin{array}{c}31960,2 \\
66316,2 \\
133402,2 \\
\end{array}$ \\
\hline $\begin{array}{lc}\text { Nouveautés } 1833 \\
-\quad 1.500 \mathrm{ex} . \\
-\quad 3.000 \mathrm{ex} . \\
-\quad 6.000 \mathrm{ex} . \\
\end{array}$ & $\begin{array}{c}4797,3 \\
77584,2 \\
241738,2 \\
\end{array}$ & $\begin{array}{c}39595,2 \\
80254,2 \\
161278,2 \\
\end{array}$ & $\begin{array}{l}15587,7 \\
33400,2 \\
67570,2 \\
\end{array}$ \\
\hline $\begin{array}{lc}\text { Nouveautés } 1840-49 \\
-\quad 1.500 \mathrm{ex} . \\
-\quad 3.000 \mathrm{ex} . \\
-\quad 6.000 \mathrm{ex} .\end{array}$ & $\begin{array}{c}43007,7 \\
193314,2 \\
505198,2 \\
\end{array}$ & $\begin{array}{c}49742,7 \\
100474,2 \\
201718,2 \\
\end{array}$ & $\begin{array}{c}47252,7 \\
96490,2 \\
193750,2 \\
\end{array}$ \\
\hline $\begin{array}{l}\text { Ensemble } 1823-49 \\
-\quad 1.500 \mathrm{ex} . \\
-\quad 3.000 \mathrm{ex} . \\
-\quad 6.000 \mathrm{ex} .\end{array}$ & $\begin{array}{c}369392,7 \\
896248,2 \\
1944382,2\end{array}$ & $\begin{array}{l}137965,2 \\
277066,2 \\
554902,2\end{array}$ & $\begin{array}{l}134995,2 \\
272314,2 \\
545398,2\end{array}$ \\
\hline
\end{tabular}

Source : nos calculs à partir des catalogues Renard et Guillaumin. * Hors imputation des frais généraux.

Pour le stock initial de 1823, plus le tirage est faible et moins les profits sont élevés pour la catégorie globale de la comptabilité. Pour les livres les plus vite vendus, la profitabilité est croissante au-delà de 3.000 exemplaires imprimés. Concernant les livres les moins vite vendus, les gains sont aussi proportionnels à la hausse du tirage. Il y aurait eu des pertes si nous avions imputé aux seules extrémités de la courbe le montant des frais généraux annuels retenus : 6.000 francs (source : Barbier, 1990, p. 263). Il faudrait enlever à ces profits bruts les impôts, frais d'annonce et avances faites aux auteurs (Cochut, dans Guillaumin, 1839, p. 1307).

Au total, la vente de livres de comptabilité est donc une bonne opération financière à long terme, car la concurrence des autres libraires est assez faible, et la fidélité des acheteurs importante en raison de la réputation de sérieux de ces entreprises. Comme la comptabilité est une matière qui évolue peu sur la période (sauf lors de l'apparition de la comptabilité industrielle), nos libraires avaient intérêt à vendre des livres tirés à 6.000 exemplaires. L'écoulement était lent, mais le rapport régulier.

Les auteurs reconnus vendent pendant très longtemps, tandis que les comptables qui vendent à compte d'auteur, ou sont moins connus, voient leurs ventes se dérouler sur une période plus courte. Renard et Guillaumin ont repris sous leurs noms plusieurs livres auparavant édités par leurs auteurs (phénomène d'autoédition propre à la spécialité comptable) : chaque auteur qui invente une 


\section{Genèse du risque éditorial : la comptabilité à l'époque romantique dans le fonds des libraires du commerce}

nouvelle méthode de transcription des comptes veut valoriser pour lui-même cette " invention » (voir tableau III supra). Ensuite, comment le libraire Guillaumin a-til dû gérer les invendus du stock Renard ? Aujourd'hui, il pourrait les solder, les détruire ou les revendre au poids du papier (Ollendorff, 2005, p. 599). A l'époque, il a certainement revendu la partie "littérature et divers " à d'autres libraires. Pour la partie commerciale " technique » il a opéré des baisses de prix entre 20 $\%$ et $80 \%$ selon la notoriété de l'auteur. Pour la partie " économie politique », il a simplement fusionné le stock de son prédécesseur et le sien. Rien ne se perd dans la chaîne des libraires-économistes, surtout s'ils sont de la même veine libérale et ont le même réseau d'amitiés politiques.

\section{Conclusion}

Dès les années 1799-1800 (an X de la première République française), il existait déjà à Paris une Librairie " économique ", sise au 117 rue de la Harpe dans l'ancien collège d'Harcourt (selon le Moniteur Universel du jeudi 8 fructidor, $p$. 1382). A cette même époque, le grand imprimeur $\mathrm{H}$. Agasse publiait un livre de calcul commercial sous la signature de M. Blanquart Sept-Fontaines ${ }^{65}$. L'idée d'un fonds spécialisé était donc dans l'air : encore fallait-il avoir les reins assez solides pour durer longtemps.

Vingt ans plus tard, vers 1820, cette idée était devenue pérenne : Bailleul ayant rencontré des difficultés avec son Journal du commerce, il trouva en son confrère Renard un repreneur. Celui-ci eut l'idée de s'allier avec les économistes libéraux et d'adosser sa librairie sur l'école spéciale de commerce qui commençait à former les comptables et les dirigeants privés de demain. Cette spécialisation était un moyen de lutter contre la baisse du prix moyen du livre qui s'amorçait déjà (Barbier, 1990). Arrivé à l'âge de la retraite, il trouva à son tour un libraire plus jeune qui s'était engagé dans la même direction que lui ; la transmission fut donc naturelle entre Renard et Guillaumin, d'où le ton d'amitié de ce dernier quand il cite son prédécesseur au début de ses catalogues.

La comptabilité et l'économie politique ont donc navigué de concert pendant toute l'époque romantique. Les économistes étaient bien conscients qu'il fallait fonder leur étude des entreprises sur des bases quantitatives solides. C'est pendant cette période que la comptabilité industrielle a connu son premier essor en France (voir les travaux des historiens français de la comptabilité : Degos, Lemarchand, Praquin, Touchelay...).

Le risque éditorial n'était pourtant pas négligeable comme nous l'avons montré. II consistait aussi bien à éviter les "rossignols » qui pesaient sur le stock, qu'à surmonter les crises économiques et commerciales qui rendaient la gestion

65. Les intérêts des comptes courans, Agasse, Paris, an X, in- $4^{\circ}, 10$ francs (livre ensuite vendu par Renard). 
quotidienne difficile. Pour cela il fallait dégager une rentabilité minimale qui dépasse les 1 ou $2 \%$ des commerces d'alors (Marco, 2009). La spécialisation dans un domaine très technique était donc un moyen de contrôler ce risque éditorial. La constitution d'un réseau d'experts (les économistes et les comptables) fut aussi un moyen efficace de se prémunir contre l'arrivée de concurrents trop pressants dans un domaine où la réputation était tout et où un auteur connu tenait le haut du pavé pendant des décennies.

La reprise du fonds Bailleul par Renard en 1820-1821 correspond bien ainsi à un moment clé de la pensée managériale : celui où l'académisme d'antan fait place à l'exubérance des projets romantiques. Avec la vente de gré à gré de ce fonds 29 ans plus tard, c'est tout un héritage intellectuel que fit fructifier Guillaumin. Et quand furent épuisés à leur tour ces vieux livres de comptabilité, le Romantisme lui-même n'y survécut pas...

\section{Bibliographie}

Barbier F. (1990), Libraires et colporteurs, dans Chartier R. et Martin H.J. (dir.), Histoire de l'édition française, Le temps des éditeurs, Du romantisme à la Belle époque, Editions du Cercle de la Librairie, Fayard, Paris.

Baudouin, fils et frères (1820), Extraits du catalogue No 36, 3 p., et manuscrit, 2 p, Paris. Diderot D. (1763), Lettre sur le commerce de la librairie, Editions Mille et Une Nuits, Paris (réédition 2003).

Fontaine de Resbecq A. de (1863), Librairie, dans Guillaumin G.U. (dir.), Dictionnaire du commerce et de la navigation, Guillaumin, Paris, t. 2 (p. 361-376).

Frégier H.A. (1849), Histoire de l'administration de la police de Paris, V-1-2, Paris.

Garnier J. (1865), Guillaumin, ses funérailles, sa vie et son œuvre, Journal des économistes, t. 45, p. 108-121, juin.

Guillaumin G.U. (1828), Catalogue de la librairie ancienne et moderne, Imprimerie de Gaultier-Laguione, Paris, in- $8^{\circ}, 8 \mathrm{p}$.

Guillaumin G.U. (1841), Catalogue, Imprimerie de Bourgogne et Martinet, Paris, in- $8^{\circ}, 16$ p.

Guillaumin G.U. (1849), Bulletin bibliographique, $n^{\circ} 1$, Paris, 8 p., octobre ; $n^{\circ} 2-3$, Paris, 24 p., novembre-décembre.

Guillaumin G.U. (1850), Bulletin bibliographique, No 4-5, Paris, 8 p., janvier-février.

Guillaumin G.U. (dir.) (1852), Dictionnaire du commerce et des marchandises, nouvelle édition, Victor Lecou, Paris (1 1 ère édition : 1837-1839).

Guillaumin G.U. (1856), Deuxième supplément au Catalogue, Typographie Simon, Raçon et compagnie, Paris, in- $8^{\circ}, 32 \mathrm{p}$.

Guillaumin G.U. (1860), Catalogue, Typographie de Crété, Corbeil, in-8º 16 p.

Guillaumin G.U. (dir.) (1863), Dictionnaire universel, théorique et pratique du commerce et de la navigation, Guillaumin et compagnie, Paris, in- $8^{\circ}, 2$ vol.

Guillaumin G.U. et Coquelin C. (dir.) (1864), Dictionnaire de l'économie politique, Guillaumin et $C^{\text {ie }}$, Paris, 2 vol., troisième édition (1ère édition : 1852-1853).

Guillaumin G.U. (1874), Catalogue, Imprimerie Viéville et Capioment, Paris, in-8 ${ }^{\circ}, 124$ p. Hatin E. (1866), Bibliographie historique et critique de la presse périodique française, Editions Anthropos, Paris (réédition 1965).

Krakovitch O. (2008), Les imprimeurs parisiens sous Napoléon $1^{\text {er }}$ : édition critique de l'enquête de décembre 1810, Paris Musées. 


\section{Genèse du risque éditorial : la comptabilité à l'époque romantique dans le fonds des libraires du commerce}

Lanzac de Laborie L. de (1910), Paris sous Napoléon : le monde des affaires et du travail, Librairie Plon, Paris.

Le Van Lemesle L. (1985), Guillaumin, éditeur d'économie politique 1801-1864, Revue d'Economie Politique, vol. 95, n² 2, p. 134-149.

Marco L. (1991), Courcelle-Seneuil, l'orthodoxe intransigeant, dans Lutfalla M. et Breton Y. (dir.), L'économie politique en France au XIX ${ }^{e}$ siècle, Economica, Paris (p. 141-161 et bibliographie p. 613-615).

Marco L. (2005), Economie politique (livres d'), dans Fouché, P., Péchoin D. et Schuwer P. (dir.), Dictionnaire encyclopédique du livre, Editions du Cercle de la Librairie, Paris (t. 2, p. 18-20, et bibliographie p. 1006).

Marco L. et Hernandez E.M. (2008), Entrepreneuriat versus salariat : construction et déconstruction d'un modèle ?, Revue française de gestion, vol. 34, No 188-189, p. 61-76, novembre-décembre.

Marco L. (2009), Histoire managériale du Bazar Bonne-Nouvelle : galeries marchandes à Paris, 1835-1863, Editions l'Harmattan, Paris, Coll. Recherches en gestion.

Mettra C. (1973), Romantisme, Encyclopédie Alpha, Editions Grange Batelière, Paris (t. 13, p. 5153-5158).

Michaud (1843), Bailleul, J.-Ch, dans Collectif, Biographie universelle ancienne et moderne, Paris (t. 2, p. 629-634).

Ollendorff M. (2005), Invendu, e, dans Fouché P., Péchoin D. et Schuwer P. (dir.), Dictionnaire encyclopédique du livre, Editions du Cercle de la Librairie, Paris (t. 2, p. 598599).

Parinet E. (2004), Une histoire de l'édition à l'époque contemporaine ( $X I X^{e}-X X^{e}$ siècle), Seuil, Coll. Points Histoire, Paris.

Périaux P. (1808), Tableau des négociants, manufacturiers, fabricants et marchands de la ville de Rouen, chez Renard (Paris) \& librairie rue Caumartin $n^{\circ} 12$, Imprimerie Périaux, Rouen.

Quérard J.M. (1842), Bailleul, Antoine ; Blanqui, Jérôme-Adolphe, dans Collectif, La littérature française contemporaine, $\mathrm{XIX}^{\mathrm{e}}$ siècle, t. 1 ${ }^{\mathrm{e}}$, Daguin, Paris (p. 126-127, p. 607).

Quillet P.H. (1823), Etat actuel de la législation sur l'administration des troupes, et particulièrement sur la solde et les traitements militaires, Antoine Bailleul, Paris (in- $8^{\circ}, x v i$ $+360+56 p)$.

Renard P.H. (1823), Catalogue des livres de commerce, Imprimerie d'Antoine Bailleul, Paris (in- $8^{\circ}, 20$ p. ; sans couverture).

Renard P.H. (1824), Catalogue des livres de commerce et autres, Imprimerie d'Antoine Bailleul, Paris (in- $8^{\circ}, 24$ p. ; couverture bleue).

Renard P.H. (1825), Notice de livres, Imprimerie Anthelme Boucher, Paris (in- $8^{\circ}, 4$ p).

Renard P.H. (1827), Catalogue des livres de commerce, Imprimerie de $\mathrm{M}^{\mathrm{e}}$ Bouquot, Troyes (in- $8^{\circ}, 32$ p. ; couverture verte).

Renard P.H. (1833), Catalogue des livres de commerce, Imprimerie de Pihan Delaforest (Morinval), Paris (in- $8^{\circ}, 40$ p. ; sans couverture).

Renard P.H. (1839), Extrait du catalogue, Imprimerie de Pollet, Soupe et Guillois, Paris (in- $8^{\circ}, 16$ p. ; sans couverture).

Renard P.H. (1840), Catalogue des livres de commerce, Imprimerie de Pollet, Paris (in- $8^{\circ}$, 32 p. ; couverture jaune).

Renouard A. (1921), Histoire de l'école supérieure de commerce de Paris, Raymond Castells, Paris (réédition 1999).

Robin C. (2000), Pratiques de gestion éditoriale, du livre à internet, Editions du Cercle de la Librairie, Paris.

Tesnière V. (2001), Le Quadrige, un siècle d'édition universitaire 1860-1968, PUF, Paris. Vroil J. de (1870), Etude sur Clicquot-Blervache, économiste du XVIII siècle, Guillaumin, Paris. 